\title{
Longitudinal transfer of rotated bunches in the CERN injectors
}

\author{
H. Timko, ${ }^{*}$ T. Argyropoulos, T. Bohl, H. Damerau, J. F. Esteban Müller, S. Hancock, and E. Shaposhnikova \\ CERN, Geneva 23, 1211, Switzerland
}

(Received 30 November 2012; published 28 May 2013)

\begin{abstract}
Bunch-to-bucket transfer between the CERN Proton Synchrotron and Super Proton Synchrotron for Large Hadron Collider-type beams is done via rotation of bunches in longitudinal phase space. For these rotated bunches, the dependence of beam transmission on bunch length had remained unexplained for a long time. Simulation-based optimization of the transfer was necessary to find the optimal rf settings for the rotation and to explain both earlier and new observations. Amongst others, we discuss why measured bunch profiles are insufficient to determine the best working point for operation. Crucially for future operation at higher intensities, we also show that the currently operational transmission can be maintained at a similar bunch length with a $40 \%$ larger longitudinal emittance, by using additional voltage from a spare cavity and optimal rotation timings.
\end{abstract}

DOI: 10.1103/PhysRevSTAB.16.051004

PACS numbers: 29.20.dk, 29.27.Ac, 29.27.Fh

\section{INTRODUCTION}

When the rf voltage is insufficient to shorten bunches adiabatically to the length required for beam transfer from one machine to another, several nonadiabatic procedures $[1,2]$ can be applied. Bunch rotation in longitudinal phase space has a broad application range [3-9]. One of the procedures uses a fast, nonadiabatic increase of the rf voltage; after $1 / 4$ of the synchrotron period, a short but unmatched bunch is extracted or recaptured with another rf voltage. However, the resulting phasespace distribution can be rather distorted as particles far from the bucket center will lag behind during the rotation.

For the Large Hadron Collider (LHC)-type beam, the difference in bucket length between the Proton Synchrotron (PS) and Super Proton Synchrotron (SPS) is reduced to a factor of 5 using higher harmonics in the PS. Even after the subsequent bunch rotation, the beam barely fits into the SPS bucket. Hence, beam transfer losses are of constant concern and posed a serious limitation a few years ago [10]. All attempts to reduce these losses by reducing the bunch length at transfer using higher voltage for the rotation failed and, at that time, it was not understood why. Earlier simulation attempts assuming different distributions were not able to reproduce the observed losses either.

The only precise longitudinal diagnostics available just before the transfer are bunch profile measurements, which can neither explain the transfer losses nor their correlation with individual bunch lengths, see Fig. 1. This is because,

\footnotetext{
*helga.timko@cern.ch

Published by the American Physical Society under the terms of the Creative Commons Attribution 3.0 License. Further distribution of this work must maintain attribution to the author(s) and the published article's title, journal citation, and DOI.
}

even if the population in the tails of the bunch was known on the percent level, the longitudinal phase-space distribution cannot be extracted from these projections (PS tomography cannot be applied at this moment, as we will discuss in Sec. III). Hence, the projection does not carry the information on what fraction of the bunch is captured by the rf bucket.

Nowadays, beam capture losses are around (5-10)\% at the currently operational intensity of about $1.6 \times$ $10^{11} \mathrm{p} /$ bunch for the LHC-type beam with $50 \mathrm{~ns}$ bunch spacing, mainly as a result of an extensive optimization of the SPS flat-bottom rf settings and reduced electroncloud activity in the machine. A more pressing issue lately is that both the PS and the SPS are at the limit of longitudinal stability already at the present intensities, and a further increase of intensity is planned for the future. The perhaps most efficient cure of these instabilities (besides impedance reduction) is to use a beam with larger longitudinal emittance. On the other hand, since the beam fills already most of the SPS bucket at injection, purely increasing the emittance will increase the losses to an unacceptable level.

To see realistic losses and explain both past and present experimental results, simulations using the measured longitudinal phase-space distribution were necessary. Through the careful modeling and subsequent experimental verification presented in this paper, we have learned that the optimization of rotated bunches cannot be based on the bunch length. In addition, we determined optimal settings for a high transmission, even at significantly larger emittances. The structure of the paper is as follows: simulation methods and the resulting longitudinal phase-space distribution are described in Sec. II; optimal rotation settings are given in Sec. III; the emittance dependence of the transmission and the bunch length is shown in Sec. IV; the effect of phase and energy errors is considered in Sec. V. 


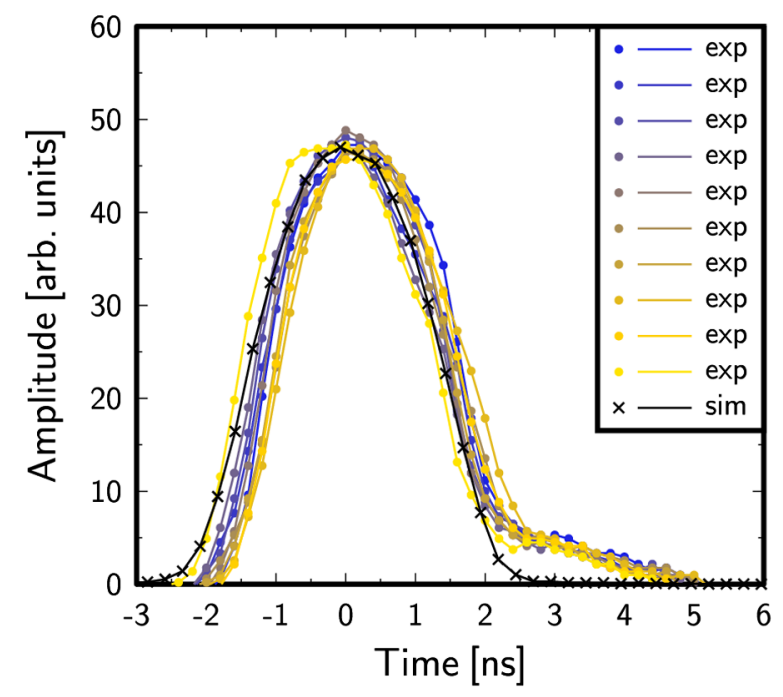

(a)

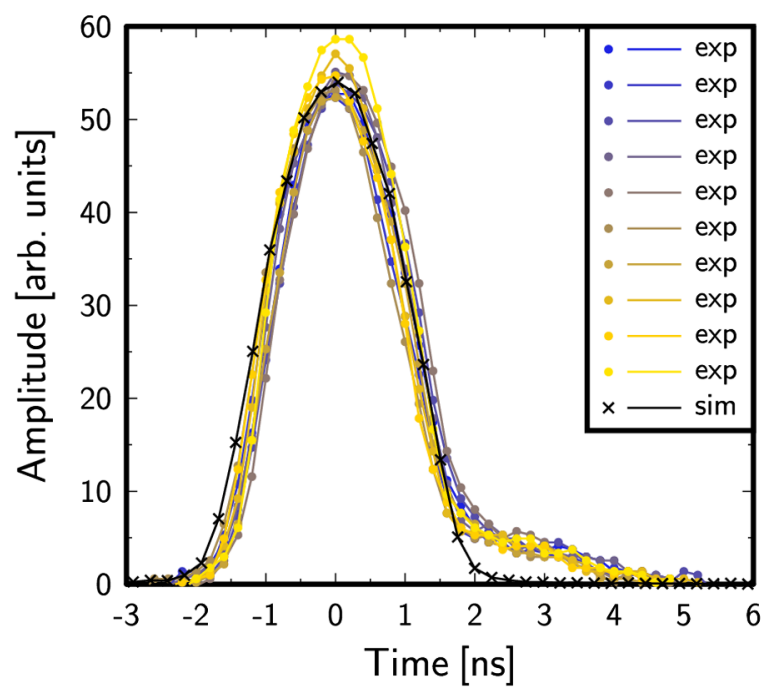

(b)

FIG. 1. Bunch profiles measured in the PS after rotation, during the last turn before extraction; the profiles of the ten first bunches in the bunch train (dots) are shown in each case. For comparison, the corresponding simulated profiles (crosses) are shown as well. The tails seen on the right-hand side are artifacts of the measurement. Part (a) shows a case with operational rotation settings, while in (b) a higher voltage has been applied to obtain a shorter bunch length. The longitudinal emittance (measured before the bunch splitting and rotation) is practically the same in both cases. Despite the shorter bunch length in (b), the transmission did not improve. The SPS bucket is $5 \mathrm{~ns}$ long.

\section{LONGITUDINAL PHASE-SPACE DISTRIBUTION OF ROTATED BUNCHES}

The rf manipulations relevant to our simulations are the following. In the PS, after arrival to flattop in the main $10 \mathrm{MHz}(h=21)$ rf system, first the bunches are lengthened adiabatically and subsequently split once or twice using the 2 nd $(20 \mathrm{MHz}, h=42)$ and 4 th $(40 \mathrm{MHz}$, $h=84$ ) harmonic rf systems. The split bunches are then shortened adiabatically in the single-rf, $40 \mathrm{MHz}$ bucket. The bunch rotation is done by first increasing rapidly the $40 \mathrm{MHz}$ rf voltage (300 kV from one cavity), and a little later the $80 \mathrm{MHz}(h=168) \mathrm{rf}$ voltage $(600 \mathrm{kV}$ from two cavities). A hot spare cavity is available for both the $40 \mathrm{MHz}$ and the $80 \mathrm{MHz}$ rf systems, and in this paper, we shall explore the different possibilities of increasing the

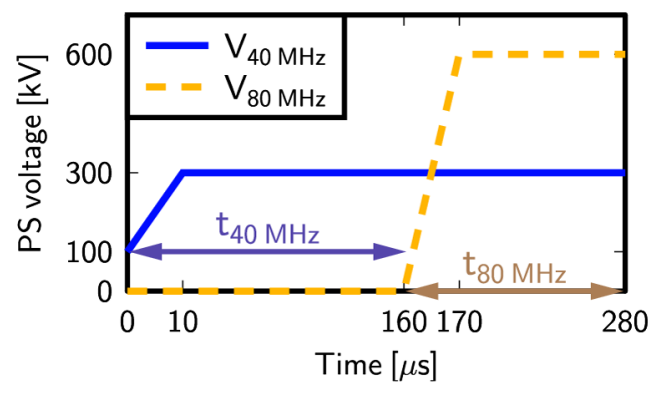

FIG. 2. Currently operational PS bunch rotation voltage program [3]. The voltage is produced with one $40 \mathrm{MHz}$ and two $80 \mathrm{MHz}$ cavities; the two rf systems are in phase. rotation voltage by using either of them in operation. The operational voltage program during the rotation, which has been optimized for $4 \mathrm{~ns}$ long bunches since several years now, is shown in Fig. 2. In the SPS, both the main $200 \mathrm{MHz}$ $(h=4620)$ and the 4 th harmonic $800 \mathrm{MHz}(h=18480) \mathrm{rf}$ systems are used throughout the whole cycle. The bunches typically spend a few seconds on the flat bottom before the start of the acceleration ramp.

Prior to all these rf manipulations, the longitudinal emittance of the bunches can be varied, if necessary, by adjusting the amount of controlled emittance blowup in the PS [11] at flat bottom and/or during the acceleration ramp.

A key feature of our approach is to use the real longitudinal phase-space distribution of bunches as an input for simulations. The distributions were measured under operational conditions in the PS at flattop via tomography $[12,13]$, which is a method for reconstructing the phasespace distribution from bunch profiles acquired during several synchrotron periods under stationary conditions. In the simulations, the measured distribution has been sampled with 500000 macroparticles and tracked through all the above-described rf manipulations starting from the double splitting(s) in the PS until the end of the flat bottom in the SPS, ${ }^{1}$ using the longitudinal tracking code ESME [15]. The tomography is taken prior to the double splitting(s) and the rotation because the quality of the measurement is the best for long bunches. Measuring rotated

\footnotetext{
${ }^{1}$ For the technical details of the tracking, see [14].
} 


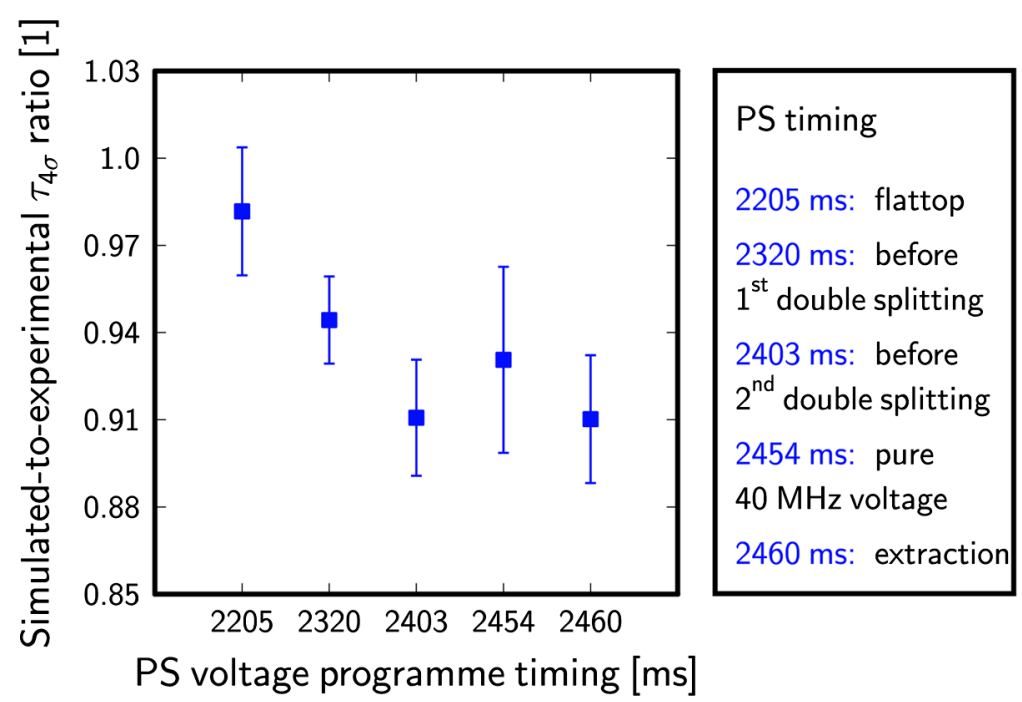

(a)

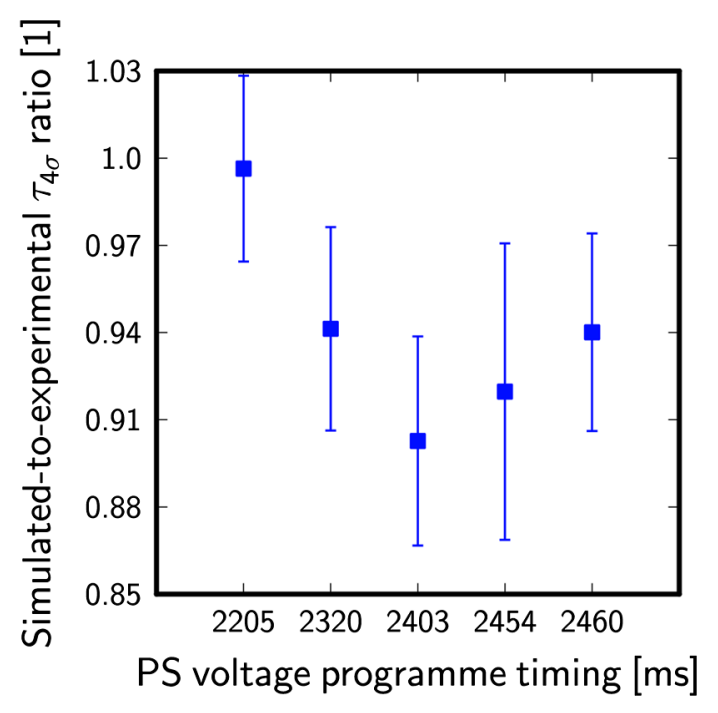

(b)

FIG. 3. Simulated-to-measured bunch length ( $4 \sigma$ Gaussian fit) ratio at different moments in the PS. A 25 ns spaced beam under operational conditions at two different intensities (measured at ejection) was investigated. (a) $6.4 \times 10^{10} \mathrm{p} / \mathrm{bunch}$. (b) $1.3 \times 10^{11} \mathrm{p} /$ bunch.



(a)

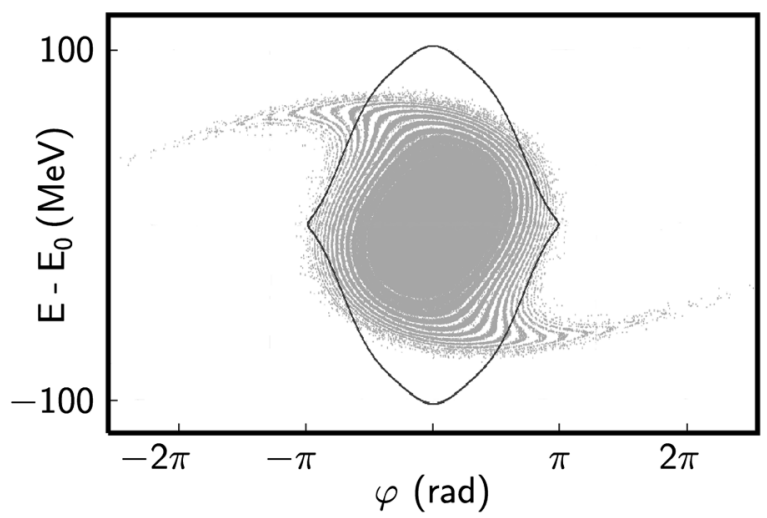

(b)

FIG. 4. Simulated bunch shapes at injection into the SPS bucket: (a) under operational conditions $\left(V_{40 \mathrm{MHz}}=300 \mathrm{kV}\right.$ and $V_{80 \mathrm{MHz}}=$ $600 \mathrm{kV}$ ); (b) using rotation voltages of $V_{40 \mathrm{MHz}}=600 \mathrm{kV}$ and $V_{80 \mathrm{MHz}}=600 \mathrm{kV}$ with optimal rotation timings. Simulations used the typical injection voltages of the SPS double-rf system, $V_{200 \mathrm{MHz}}^{\mathrm{SPS}}=2 \mathrm{MV}$ and $V_{800 \mathrm{MHz}}^{\mathrm{SPS}}=0.34 \mathrm{MV}$ in phase. Note that the empty regions in the distribution are due to the filamentation of the bunch during the uncontrolled emittance blowup.

bunches via tomography results in an unsatisfactory quality not only due to the short bunch length, but more importantly, due to the lack of information (originating from the limited acquisition time). Hence, without simulations we are "blind" in some sense, see Fig. 1.

First simulations were not able to reproduce the experimentally observed bunch lengths at PS extraction. Hence, we compared the simulated and the measured bunch lengths at different moments during the PS flattop, see Fig. 3. Since the measurements were averaged over $10-15$ acceleration cycles, but the simulations over $2-4$ bunches (whose distributions represented best the average), there is some scatter in the ratio of the two. The ratio at the moment of the tomography measurement (2205 ms) is therefore not exactly one either. ${ }^{2}$ However, it can be concluded that on average there is no significant intensity dependence and that the measured bunches are longer already before any rf voltage manipulations are applied. In the identified time span at PS flattop, between the tomography measurement and the double splitting(s), the bunches are synchronized with respect to the reference phase of the SPS in preparation of the beam transfer. The

\footnotetext{
${ }^{2}$ Note that the data for tomographic reconstruction can be acquired only for one bunch out of the bunch train, while bunch profiles and bunch lengths are measured for all the bunches.
} 
locking process of the synchronization loop introduces a phase error between bunch and bucket, which might well lead to the uncontrolled longitudinal emittance blowup observed. As a simple model of this effect, an artificial blowup was included in the simulations by introducing a phase error between the bucket center and the center of the bunch and then keeping the voltage constant until the filamentation was complete. The time available for the filamentation (around 20 synchrotron periods $T_{s}$ ) is sufficiently long to reach full equilibrium (which takes about $10 T_{s}$ ). The phase error was chosen such that simulated and experimentally observed bunch lengths would match.
We have mentioned that, for rotated bunches, decreasing the bunch length does not necessarily improve the transmission. On the contrary, when optimizing the rotation timings, the transmission can sometimes actually be improved by lengthening the bunches (as will be shown later in Fig. 6, squares and triangles). This result can be understood by looking at the longitudinal phase-space distribution of the bunches, see Fig. 4. Indeed, we achieve to rotate relatively more particles into the core of the bunch and reduce the number of particles in the tails. As a result, the bunch length increases, but more particles are captured in the injection bucket and the losses are reduced.

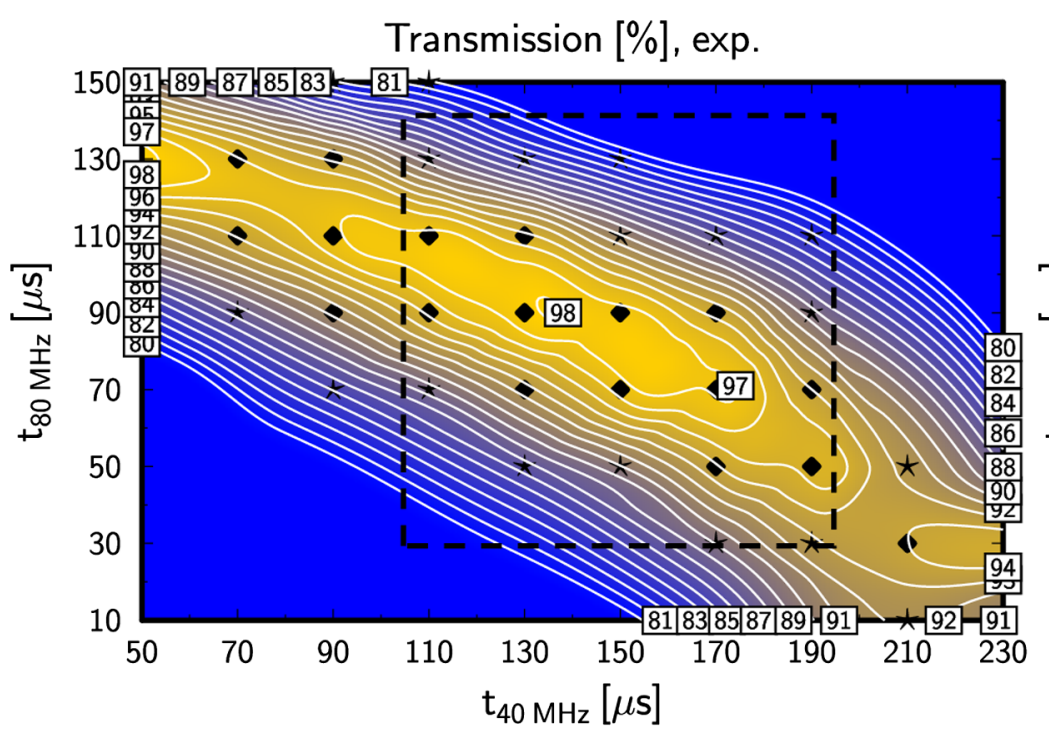

(a)

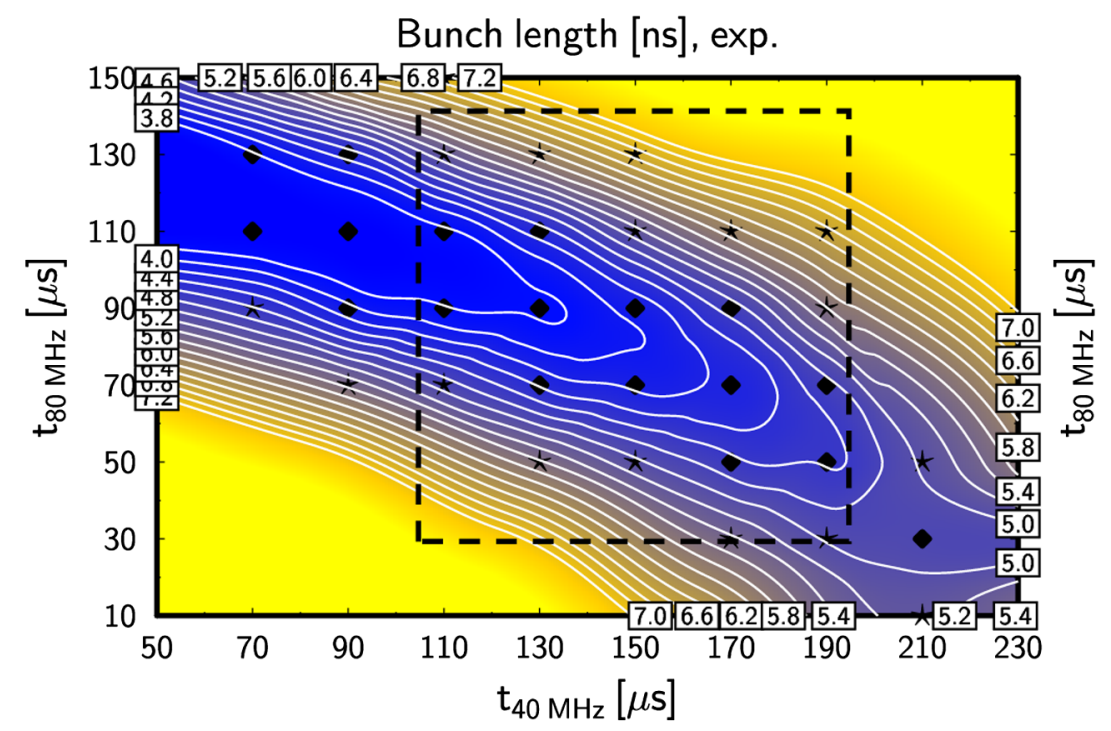

(c)

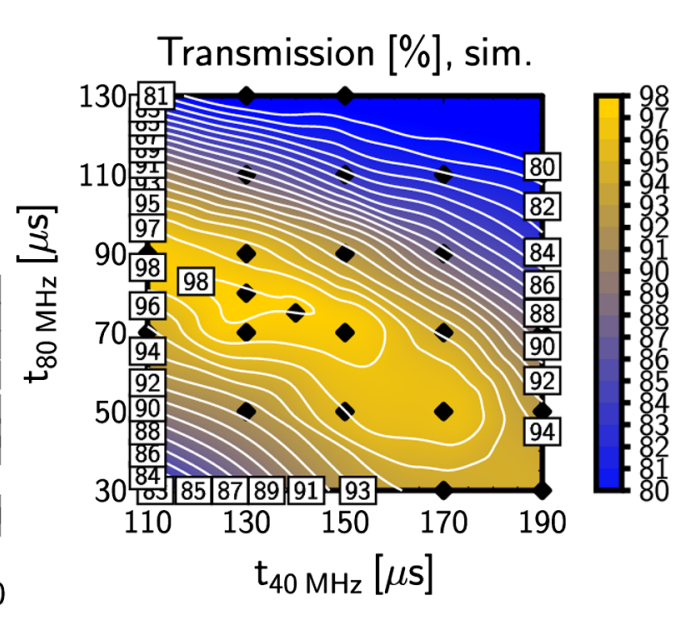

(b)

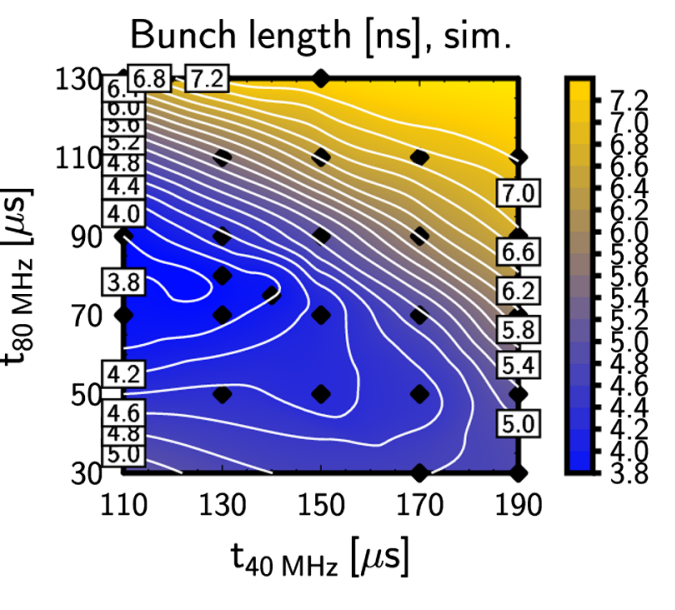

(d)

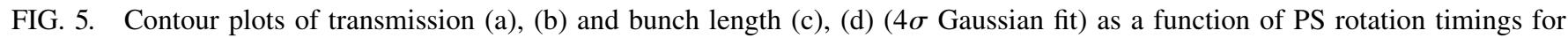
$V_{40 \mathrm{MHz}}=600 \mathrm{kV}$ and $V_{80 \mathrm{MHz}}=600 \mathrm{kV}$. Measured and simulated results are shown in parts (a), (c) and (b), (d), respectively. In (a) and (c), measurements points marked with stars are averages of five measurements, while diamonds mark an average of ten; the boxes mark the region that was also simulated. Measurements were done with a $50 \mathrm{~ns}$ spaced beam at an intensity of $1.6 \times 10^{11} \mathrm{p} / \mathrm{bunch}$. 
In reality, the machine impedance will change the potential well seen by the particles in the bucket. Hence, the synchrotron frequency distribution of the particles, and therefore also the rotated bunch shape, will be affected at high intensities. Even though our present tracking does not take intensity effects into account, the measured phasespace distribution that is used in the simulations does; a model taking intensity effects fully into account is under way. However, since our present, idealized simulations are in good agreement with the measurement results up to now, a low-intensity approximation appears to be still adequate.

\section{SIMULATION-BASED OPTIMIZATION}

As we have seen, the rotated bunches have an " $S$-shaped" distribution in phase space. Hence, even at perfect injection, some parts of the bunch will not be captured in the buckets. The distribution of the bunches could in principle be improved by performing the rotation in a lower-harmonic system that provides a more linear voltage in the region occupied by the bunch. However, the required bunch spacings do not allow us to do so; only in the case of the $50 \mathrm{~ns}$ spacing, an rf voltage at $20 \mathrm{MHz}$ could be used, but the existing cavities cannot provide enough voltage for the rotation.

On the other hand, already by just optimizing the timings $t_{40 \mathrm{MHz}}$ and $t_{80 \mathrm{MHz}}$ (see Fig. 2) of the rotation, the elongation of the tails can be reduced. In addition, using higher voltage for the rotation, which is potentially available in the PS from spare cavities, the rotation can be made more linear. With systematic simulation scans of the rotation timings, we have identified the optimal settings for the different rotation voltage options. They were later also confirmed by measurements using a test cycle with a shorter-than-operational flat bottom in the SPS and the operational PS cycle. Both for losses and emittance (for the emittance dependence, see the next section), the best result can be achieved by using the spare $40 \mathrm{MHz}$ cavity operationally, increasing thereby the rotation voltage to $V_{40 \mathrm{MHz}}=600 \mathrm{kV}$ and keeping $V_{80 \mathrm{MHz}}=600 \mathrm{kV}$. In this case, the optimal timings found in simulation are $t_{40 \mathrm{MHz}}=130 \mu \mathrm{s}$ and $t_{80 \mathrm{MHz}}=80 \mu \mathrm{s}$ and in experiments $t_{40 \mathrm{MHz}}=130 \mu \mathrm{s}$ and $t_{80 \mathrm{MHz}}=90 \mu \mathrm{s}$, see Fig. 5. With these settings, simulations predict that the losses can be reduced from $4.4 \%$ to $1.9 \%$ compared to the operational rotation. In measurements at $1.6 \times 10^{11} \mathrm{p} /$ bunch with a $50 \mathrm{~ns}$ spaced beam, a loss reduction from $5.2 \%$ to $2.3 \%$ was achieved, in good agreement with the predictions.

Both in simulations and experimentally, the bunch length was obtained from a $4 \sigma$ Gaussian fit to the bunch profiles at PS extraction. The transmission was measured in the SPS with a beam current transformer as the ratio of beam intensity at $30 \mathrm{GeV} / c$ (the flat-bottom momentum is $26 \mathrm{GeV} / c$ ) to injected intensity (measured after $20 \mathrm{~ms}$ ). The beginning of the acceleration ramp is included to take all capture losses into account. In simulations, it is sufficient to consider the fraction of particles that are outside the buckets at the end of the flat bottom.

\section{EMITTANCE DEPENDENCE}

With the LHC requesting higher-intensity beams and both the PS and the SPS being at the limit of stability, beam instabilities have become one of the biggest

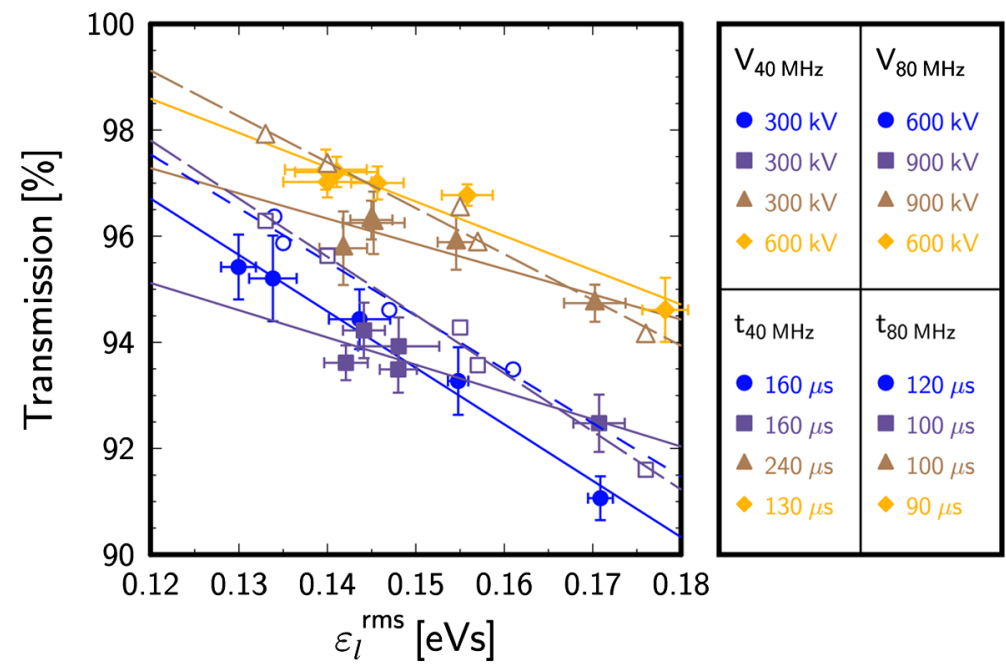

(a)

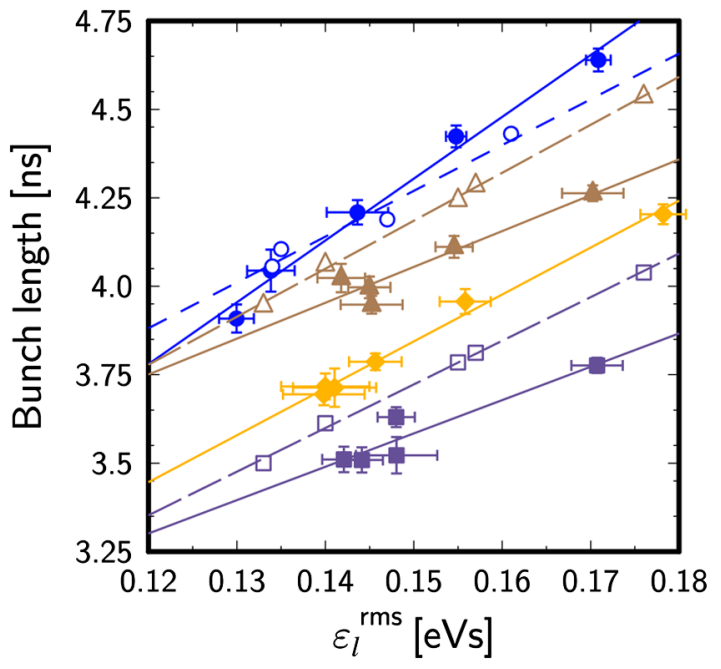

(b)

FIG. 6. Emittance dependence of transmission (a) and bunch length (b). Full and empty markers (continuous and dashed lines) mark experimental and simulation results, respectively. The blue circles correspond to currently operational settings, the violet squares to earlier optimization attempts based on bunch length. The brown triangles and the yellow diamonds show the results using optimized timings and additional $V_{80 \mathrm{MHz}}$ or $V_{40 \mathrm{MHz}}$, respectively, for the rotation. No simulations were done for the latter case. The bunch length is obtained from a $4 \sigma$ Gaussian fit in all cases. 
concerns; efforts are ongoing to identify the source of these instabilities [16,17]. Increased longitudinal emittance is one of the most efficient cures applicable. Hence, knowing how transmission and bunch length change with increased longitudinal emittance is essential, see Fig. 6.

The currently operational curves of transmission and bunch length as a function of longitudinal emittance are marked with full circles. The bunches were then shortened by applying additional voltage from the spare $80 \mathrm{MHz}$ cavity and adjusting $t_{80 \mathrm{MHz}}$ to obtain the shortest possible bunch length (full squares in the figure). As a result, significantly shorter bunch lengths [(10-15)\%] were achieved for all emittances, however, the transmission remained unchanged. Simulations reproduced these results (corresponding empty markers) and explained them with losses from the elongated parts of the bunch.

An improved transmission can be obtained when using the additional $80 \mathrm{MHz}$ rf voltage with the optimal timings found in simulations, while the bunch lengths remain similar to the currently operational values (see triangles). This demonstrates that the simulation-based optimization works for these parameters. However, losses will still be a problem for the injection of bunches with much larger emittance into the 5 ns SPS buckets with an operational safety margin. In the preferred scenario that uses the additional $40 \mathrm{MHz}$ voltage with optimized timings (diamond markers), not only the transmission can be improved, but bunches can in addition be made shorter. This has the advantage that even with $40 \%$ larger emittance, the currently operational transmission can be maintained, at a similar bunch length.

The emittance scans were carried out with the experimentally found optimal timings written in Fig. 6. The optimal timings are slightly different in simulation. Furthermore, the measured and simulated slopes for both transmission and bunch length are not exactly the same either. These differences are attributed to the fact that simulations did not take intensity effects into account. In addition, with a spare cavity on, occasional longitudinal instability was observed in the PS, which is why measurements around (0.14-0.15) eVs have a large spread and below $0.14 \mathrm{eVs}$ were not possible at all. Despite the idealized simulations, the results match remarkably well. However, at even higher intensities, intensity effects should be included.

\section{PHASE AND ENERGY ERRORS}

All simulation results presented above assumed perfect injection into the SPS bucket, i.e., the center of mass of the bunch is placed into the center of the bucket. On the other hand, in the real machine, there are phase and/or energy errors between the bunch and the rf bucket at injection. Phase errors at injection, for instance, are corrected by the SPS phase loop for some bunches, but not for all the bunches, since beam loading modifies the stable position of the bunches.

The effect of realistic phase and/or energy errors of up to $200 \mathrm{ps}$ and/or $10 \mathrm{MeV}$, respectively, is shown in Fig. 7. Even for the maximum values, the difference in the transmission is small and would be at most of the order of the measurement error. Interestingly, not only the separate effect of the phase or energy error is small, but also the combined phase and energy error results in a similar, or sometimes even smaller, reduction of the transmission. This is again explained by the longitudinal

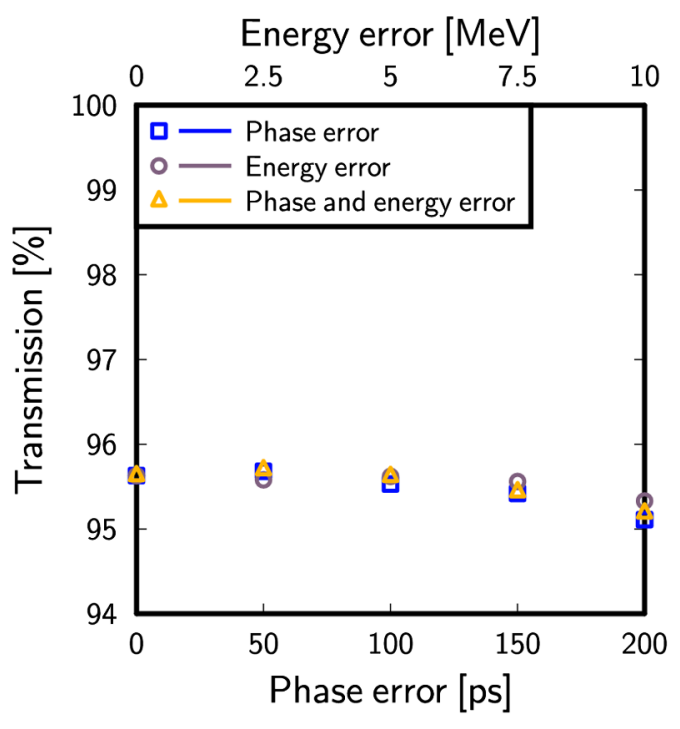

(a)

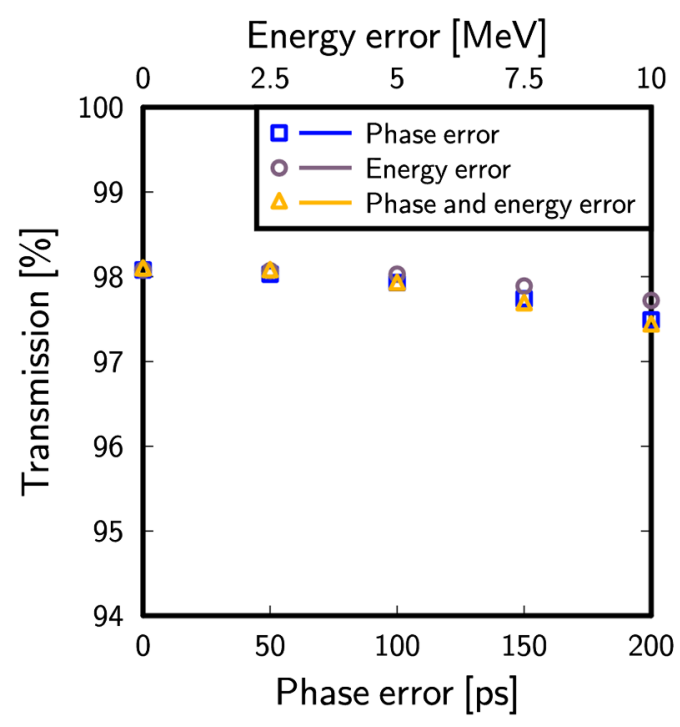

(b)

FIG. 7. Phase and/or energy errors: (a) under operational conditions; (b) using rotation voltages of $V_{40 \mathrm{MHz}}=600 \mathrm{kV}$ and $V_{80 \mathrm{MHz}}=$ $600 \mathrm{kV}$ with optimal rotation timings. 
phase-space distribution of the rotated bunches: since the losses are dominated by losses from the tails, a mismatch between the bunch and the bucket will create more losses from one tail but reduce the losses from the other tail.

\section{CONCLUSIONS}

During the rotation in the PS, the bunches extend to the nonlinear region of the $\mathrm{rf}$ buckets, which results in a longitudinally unmatched, " $S$-shaped" phase-space distribution. Hence, seemingly intuitive scaling laws for beam capture often do not apply for these bunches. To identify the optimal settings for such a rotation, simulation-based optimization turned out to be indispensable. The key to understand earlier and present measurements results, at least in the case of the PS-to-SPS transfer, was to use the real bunch distribution (measured prior to the rotation) as a starting point for the simulations. Matching the experimentally observed bunch length was also an important ingredient.

The ideal settings predicted by simulations have been confirmed by reproducible, systematic measurement scans using a test cycle in the SPS. By applying additional voltage from the spare $40 \mathrm{MHz}$ cavity and optimized rotation timings, the currently operational transmission was maintained with $40 \%$ larger longitudinal emittance, at a similar bunch length. The validation and operational use of these improved settings is delayed by repair works on a faulty accelerating cavity. The increase in emittance that these new settings promise to allow for is of great importance for the future high-intensity LHC operation, for which at the present longitudinal instabilities in the PS and SPS are one of the major intensity limitations.

Based on the obtained results, we propose to use in the future a higher voltage for the bunch rotation in the PS. The voltage could be increased either by using the currently operational cavities or by using the hot spare cavity operationally. In both cases, an upgrade of the power supplies would be desirable.

\section{ACKNOWLEDGMENTS}

Support for our machine development studies from the CERN PS and SPS operation teams is gratefully acknowledged.
[1] S. Y. Lee, Accelerator Physics (World Scientific, London, 2004), 2nd ed.

[2] D. Boussard, in Proceedings of the CAS-CERN Accelerator School: Antiprotons for Colliding-beam Facilities, Geneva, Switzerland (CERN, Geneva, 1984), pp. 261-290.

[3] R. Garoby, CERN Technical Report No. PS/RF/Note 9317, 1993.

[4] A. Faugier, CERN Technical Report No. CERN-SPS-AOPAF-jf (CERN-SPS-Improvement-Report-177), 1980.

[5] E. Adli, W. An, R. Assmann, R. Bingham, A. Caldwell, S. Chattopadhyay, N. Delerue, F. M. Dias, I. Efthymiopoulos, E. Elsen et al., CERN Technical Report No. CERN-SPSC2011-020, SPSC-I-240, 2011.

[6] W. Kriens, in Proceedings of the 17th Particle Accelerator Conference, Vancouver, BC, Canada, 1997 (IEEE, New York, 1997), pp. 231-233.

[7] I. Kourbanis and D. Wildman, in Proceedings of the 18th Particle Accelerator Conference, New York, 1999 (IEEE, New York, 1999), p. 2840.

[8] D.-Y. Yin, Y. Liu, Y.-J. Yuan, J.-C. Yang, P. Li, L.-R. Mei, W.-P. Chai, and X.-H. Zhang, Chinese Phys. C 36, 552 (2012) [http://stacks.iop.org/1674-1137/36/i=6/a=012].

[9] N.A. Tahir, S. Udrea, C. Deutsch, V.E. Fortov, N. Grandjouan, V. Gryaznov, D. H. H. Hoffmann, P. Hülsmann, M. Kirk, I. V. Lomonosov et al., Laser Part. Beams 22, 485 (2004) [http://dx.doi.org/10.1017/S0263034604040145].

[10] E. Shaposhnikova, T. Bohl, T.P. R. Linnecar, and J. Tückmantel, in Proceedings of the 9th European Particle Accelerator Conference, Lucerne, 2004 (EPS-AG, Lucerne, 2004), pp. 1906-1908.

[11] D. Boussard, CERN Technical Report No. CERN-SPS-857-ARF, 1985.

[12] J.-F. Comblin, S. Hancock, and J.-L. Sanchez Alvarez, Revised Technical Report No. PS/RF/Note 2001-010, 2004.

[13] http://tomograp.web.cern.ch/tomograp/publications.html.

[14] H. Timko, T. Argyropoulos, T. Bohl, H. Damerau, J.F. Esteban Müller, S. Hancock, and E. Shaposhnikova, CERN Technical Report No. CERN-ATS-Note-2012-059 PERF, 2012.

[15] The ESME code was developed at Fermilab by James MacLachlan and co-workers [http://www-ap.fnal.gov/ ESME/].

[16] H. Damerau, S. Hancock, and M. Schokker, in Proceedings of the 46th ICFA Advanced Beam Dynamics Workshop on High-Intensity and High-Brightness Hadron Beams (ICFA, Morschach, 2010), MOPD52.

[17] T. Argyropoulos, T. Bohl, J. E. Müller, E. Shaposhnikova, H. Timko, and C. Bhat, in Proceedings of the 3rd International Particle Accelerator Conference, New Orleans, Louisiana, USA, 2012 (IEEE, Piscataway, NJ, 2012), WEPPR061. 